\title{
Gesundheitsausgaben: Schweiz wiederum im zweiten und dritten Rang
}

\author{
Im Vergleich zum Bruttoinlandsprodukt hat die Schweiz seit 1999 nach den \\ USA die zweithöchsten Gesundheitsausgaben der Welt. Die Pro-Kopf-Aus- \\ gaben für das Gesundheitssystem werden nur von den USA und Norwegen \\ übertroffen. Die OECD hat auf einer CD-ROM zahlreiche weitere Daten bis \\ 2005 über die Gesundheitssysteme von 30 Ländern publiziert.
}

Gerhard Kocher

Korrespondenz:

Dr. rer. pol. Gerhard Kocher Haldenweg 10 a CH-3074 Muri b. Bern Tel. 0319526707

kocher@spectraweb.ch
Die aktuelle Jahres-CD-ROM der OECD (Organisation für wirtschaftliche Zusammenarbeit und Entwicklung in Paris) enthält zahlreiche Statistiken zu den Gesundheitswesen der 30 OECD-Mitgliedsländer [1]. Die neusten Zahlen betreffen meist das Jahr 2005, viele Datenreihen gehen zurück bis 1960. Die interaktive CD erlaubt aufschlussreiche Vergleiche zwischen den Industrieländern. Allerdings sind wegen unterschiedlicher Definitionen und Gesundheitssysteme nicht alle Daten bis auf Kommastellen vergleichbar. Zudem enthalten die Statistiken teilweise Schätzungen.

\section{Gesundheitsausgaben als BIP-Anteil: Rang 2 für die Schweiz}

2005 beliefen sich die Gesamtgesundheitsausgaben der Schweiz auf 11,6\% des Bruttoinlandsprodukts BIP (Tab. 1). Höher waren die Ausgaben nur in den USA (15,3\%). Die Schweiz ist seit 1999 immer im zweiten Rang nach den USA. Der OECD-Durchschnitt beträgt 9\%. Die USA geben $70 \%$ und die Schweiz 29\% mehr aus. Die Kostenentwicklung von 2000 bis 2005 ist trotz zahlloser Sparversuche immer noch stark: 2000 gaben die OECD-Länder im Schnitt 8\% aus, 2005 schon 9\%. In sämtlichen Ländern ist der BIP-Anteil in diesem Zeitraum gestiegen, durchschnittlich um 13,1\% (Schweiz 11,5\%, USA 15,9\%).

Diese Rangliste des BIP-Anteils hängt selbstverständlich nicht nur von den nationalen Gesundheitsausgaben (Zähler) ab, sondern auch von der Wirtschaftsentwicklung (Nenner), die im BIP zum Ausdruck kommt. Die Schweiz erzielte zwischen 2000 und 2005 ein BIP-Wachstum von nur gerade 1,9\% pro Jahr (OECD-Durchschnitt 6,2\%). Dies entspricht dem 27. Rang von 30 Ländern. Um ein zutreffendes Bild zu erhalten, muss deshalb ein zweites Ranking beachtet werden, das direkt und ausschliesslich die Gesundheitsausgaben darstellt.
Dépenses de santé:

\section{la Suisse à nouveau au deuxième et troisième rang}

Le nouveau CD-ROM de l'Organisation de coopération et de développement économiques (OCDE) à Paris contient de nombreuses statistiques sur la santé publique des 30 pays membres [1]. Les chiffres les plus récents du disque sont tirés de l'année 2005 et de nombreux groupes de données remontent à 1960. Le CD-ROM interactif permet de tirer des comparaisons intéressantes entre les pays industrialisés. Cela dit, les données ne sont pas en tous points comparables en raison de définitions différentes et des systèmes de santé particuliers à chaque pays. De plus, les statistiques contiennent parfois des estimations.

Par rapport au produit intérieur brut, la Suisse a, depuis 1999 , les dépenses de santé les deuxièmes plus hautes du monde après les Etats-Unis. Les dépenses du système de santé par habitant ne sont dépassées que par les Etats-Unis et la Norvège.

\section{Gesundheitsausgaben pro Einwohner: Rang 3 für die Schweiz}

Gemäss OECD gab die Schweiz 2005 pro Kopf US-\$ 4177 für das Gesundheitswesen aus (Tab. 2).

Tabellenerster sind wie bisher die USA mit US-\$ 6401 (53\% höher als die Schweiz). Norwegen ist mit US-\$ 4354 wie schon 2003 auf dem zweiten Platz. Die Schweizer Ausgaben sind 55\% höher als der OECD-Durchschnitt von US-\$2700, die USA-Ausgaben sind 137\% höher. Alle diese Dollarwerte sind kaufkraftbereinigt; sie berück- 
sichtigen also die unterschiedliche Kaufkraft der Länder. Zum Wechselkurs gerechnet wäre die Schweiz 2005 ebenfalls an dritter Stelle mit US-\$5694, nach den USA (6401) und Norwegen (5912). Unsere Pro-Kopf-Ausgaben sind bei diesem Vergleich nur 8,9\% geringer als die der USA.

Kein einziges Land konnte zwischen 2000 und 2005 die Pro-Kopf-Ausgaben senken oder einfrieren. Überall stiegen sie um mindestens 16\% (Island) bis höchstens 91,1\% (Slowakische Republik). Das Durchschnittswachstum betrug 37\% (Schweiz 31,3\%, USA 40,1\%, Deutschland $24,8 \%$ ). Die von der britischen Regierung gewollten Mehrinvestitionen in den National Health Service NHS führten zu einer starken Ausgabensteigerung von $46,5 \%$.

Seit 1961 jährlich 7,6\% mehr Ausgaben Ein Blick auf den Zeitraum 1961 bis 2005 zeigt, dass das durchschnittliche Ausgabenwachstum in der Schweiz pro Kopf und Jahr 7,6\% beträgt.

\section{Tabelle 1}

Nationale Gesundheitsausgaben 2005 in \% des Bruttoinlandsprodukts BIP.

\begin{tabular}{lccc}
\hline Land & \% BIP & Rang & Rang \\
& $\mathbf{2 0 0 5}$ & $\mathbf{2 0 0 5}$ & $\mathbf{2 0 0 0}$ \\
\hline USA & 15,3 & 1 & 1 \\
\hline Schweiz & $\mathbf{1 1 , 6}$ & $\mathbf{2}$ & $\mathbf{2}$ \\
\hline Frankreich & 11,1 & 3 & 5 \\
\hline Deutschland & 10,7 & 4 & 3 \\
\hline Belgien & 10,3 & 5 & 8 \\
\hline Portugal & 10,2 & 6 & 7 \\
\hline Österreich & 10,2 & 6 & 4 \\
\hline Griechenland & 10,1 & 7 & 6 \\
\hline Kanada & 9,8 & 8 & 7 \\
\hline Island & 9,5 & 9 & 6 \\
\hline Dänemark & 9,1 & 10 & 10 \\
\hline Norwegen & 9,1 & 10 & 9 \\
\hline Schweden & 9,1 & 10 & 9 \\
\hline Neuseeland & 9 & 11 & 12 \\
\hline Italien & 9,9 & 12 & 11 \\
\hline Grossbritannien & 8,3 & 13 & 13 \\
\hline Spanien & 8,2 & 14 & 14 \\
\hline Türkei & 7,6 & 15 & 15 \\
\hline Irland & 7,5 & 16 & 17 \\
\hline Finnland & 7,5 & 16 & 15 \\
\hline Tschechische Republik & 7,2 & 17 & 16 \\
\hline Slowakei & 7,1 & 18 & 19 \\
\hline Mexiko & 6,4 & 19 & 18 \\
\hline Polen & 6,2 & 20 & 20 \\
\hline Südkorea & 21 & 21 \\
\hline Durchschnitt & & & \\
\hline & 9,9 & & \\
\hline
\end{tabular}

Grösste Unterschiede zeigen sich allerdings bei der Betrachtung nach 5-Jahres-Perioden. Von 1961 bis 1965 stiegen die Gesundheitsausgaben jährlich um 8,7\%. Es folgten die zehn Jahre mit der höchsten «Kostenexplosion»: 1966-1970 mit $12,8 \%$ und 1971-1975 mit 14,7\% pro Jahr. Die weitere Entwicklung: 1976-1980 5,1\%, 19811985 6,9\%, 1986-1990 7,4\% und 1991-2000 $6 \%$. «Nur» noch um 3,7\% stiegen die Pro-KopfKosten jährlich zwischen 1996 und 2000, um 4,1\% zwischen 2001 und 2005.

Ganz anders verlief die Ausgabenentwicklung im Vergleich zum Bruttoinlandsprodukt. Die jährliche Abnahme (!) von 1961 bis 1965 betrug $1,2 \%$. In den folgenden 5-Jahres-Perioden unterschieden sich die Zunahmen bis fast zum Fünffachen: $3,7 \%, 5 \%, 1,1 \%, 1,1 \%, 1,3 \%, 3,2 \%$ und $1,4 \%$. Von 2000 bis 2005 stieg der BIP-Anteil jährlich um 2,2\%.

Das Bundesamt für Statistik [2] prognostiziert übrigens die Gesundheitskosten für 2007 auf 57,120 Mrd. Franken (Vertrauensintervall

\section{Tabelle 2}

Gesundheitsausgaben pro Kopf 2005 in US-\$ (kaufkraftbereinigt).

\begin{tabular}{|c|c|c|c|}
\hline Land & $\begin{array}{l}\text { US-\$ } \\
2005\end{array}$ & $\begin{array}{l}\text { Rang } \\
2005\end{array}$ & $\begin{array}{l}\text { Rang } \\
2000\end{array}$ \\
\hline USA & 6401 & 1 & 1 \\
\hline Norwegen & 4364 & 2 & 3 \\
\hline Schweiz & 4177 & 3 & 2 \\
\hline Österreich & 3519 & 4 & 5 \\
\hline Island & 3443 & 5 & 4 \\
\hline Belgien & 3389 & 6 & 10 \\
\hline Frankreich & 3374 & 7 & 8 \\
\hline Kanada & 3326 & 8 & 7 \\
\hline Deutschland & 3287 & 9 & 6 \\
\hline Dänemark & 3108 & 10 & 9 \\
\hline Griechenland & 2981 & 11 & 13 \\
\hline Irland & 2926 & 12 & 15 \\
\hline Schweden & 2918 & 13 & 11 \\
\hline Grossbritannien & 2724 & 14 & 14 \\
\hline Italien & 2532 & 15 & 12 \\
\hline Neuseeland & 2343 & 16 & k. A. \\
\hline Finnland & 2331 & 17 & 16 \\
\hline Spanien & 2255 & 18 & 18 \\
\hline Portugal & 2033 & 19 & 17 \\
\hline Tschechische Republik & 1479 & 20 & 19 \\
\hline Südkorea & 1318 & 21 & 20 \\
\hline Slowakei & 1137 & 22 & 21 \\
\hline Polen & 867 & 23 & 22 \\
\hline Mexiko & 675 & 24 & 23 \\
\hline Türkei & 586 & 25 & 24 \\
\hline Durchschnitt & 2700 & & \\
\hline
\end{tabular}


55,271 bis 59,036 Mrd.) und für 2008 auf 59,338 (56,994 bis 61,784 Mrd.).

\section{Hoher Selbstzahleranteil und weitere Schweizer Spezialitäten}

Direkt aus dem eigenen Geldbeutel zahlen die Schweizer im Schnitt US-\$ 1739 (2005). Unter den 22 OECD-Ländern, für die die Zahlen dieser sogenannten Out-of-pocket-Ausgaben vorliegen, ist dies bei weitem der höchste Betrag. Er ist 3,6mal höher als der Durchschnitt und besteht vor allem aus den Zahlungen für die Zahnmedizin, nicht kassenpflichtige Medikamente, Pflegeheime und Spitex sowie Franchisen und Selbstbehalten. Die Gesamtsumme der Selbstzahlungen in der Schweiz (Fr. 16,163 Mrd.) macht 30,5\% der nationalen Gesamtausgaben aus.

Die sehr hohe Direktbelastung der Privathaushalte ist eine traditionelle Besonderheit unseres Landes und wäre in den meisten anderen Staaten aus politischen Gründen nicht denkbar. Was bei uns weite Kreise (nicht nur die Rechte) als «Selbstverantwortung» preisen, wird in anderen Ländern als asozial und als Bestrafung der Kranken (victim blaming) tabuisiert. Neben dem Nichteinschluss der Zahnmedizin in die Krankenversicherung, den einkommensunabhängigen Krankenversicherungsprämien, der Selbstdispensation, den HMOs und dem Extremföderalismus ist die hohe Direktbelastung im internationalen Vergleich eine der grössten Anomalien unseres Gesundheitssystems.

\section{Medikamentenausgaben pro Kopf: Schweiz an der Spitze}

Die OECD berechnet den Arzneimittelabsatz pro Kopf in der Schweiz (Tab. 3) auf US-\$ 560 (2005). Dies ist $64 \%$ höher als der Durchschnitt der erfassten 20 Länder. Von 2000 bis 2005 stiegen diese Ausgaben in der Schweiz um 35\%, im Schnitt der 20 Länder um 70\%. In dieser Periode nahmen die Medikamentenausgaben pro Kopf in allen Ländern zu (Ausnahme: Japan mit Nullwachstum). In der jeweiligen Landeswährung betrug das durchschnittliche Wachstum in diesen fünf Jahren $62 \%$. Die geringste Zuwachsrate hatte hier die Schweiz (+ 3,1\%).

Der Arzneimittelabsatz 2005 in der Schweiz betrug 5,208 Mrd. Franken (2000: 5,053 Mrd.).

Allerdings weisen die OECD und das Bundesamt für Statistik seit 2002 einen zu geringen Medikamentenabsatz aus. Grund: Die Medikamentenausgaben der Spitäler von über 1 Milliarde Franken sind in den obigen Zahlen nicht enthalten. Fridolin Marty [3] kommt für 2005 auf totale Medikamentenkosten inklusive Spitälern von 6,269 Mrd. Franken (rund 12\% aller Gesund- heitsausgaben). Die Pharmaindustrie übrigens operiert bei den «Medikamentenausgaben» fast nur mit den Herstellerabgabepreisen, was selbstverständlich irreführend ist (entscheidend sind die Publikumspreise) und viel zu niedrige Werte ergibt (2005: Fr. 4,194 statt 6,269 Mrd.).

\section{Hohe und wachsende Ärztedichte in der Schweiz}

Die Zahl der berufstätigen Ärzte in unserem Land ist hoch: 3,8 Ärzte pro 1000 Einwohner. In der OECD-Rangliste 2005 ist die Schweiz zusammen mit Italien und Spanien damit im 2. Rang von 22 erfassten Ländern (Tab. 4). Die Dichte ist $27 \%$ höher als der OECD-Durchschnitt. Auffallend ist der grosse Unterschied zu Ländern wie Kanada, USA und Grossbritannien (mit 2,2 bzw. 2,4 berufstätigen Ärzten je 1000 Einwohner). Seit 2000 ist die Schweizer Ärztedichte um 8,6\% gestiegen, der Durchschnitt der 22 Länder um 7,2\%.

\section{Lange Aufenthaltsdauer in Akutspitälern}

Ein altes Problem ist die (zu) lange Verweildauer in unseren Spitälern, auch wenn sie im Rückgang begriffen ist und durch die SwissDRGs noch weiter reduziert werden wird. 2005 waren wir bei

\section{Tabelle 3}

Arzneimittelabsatz in US-\$ pro Kopf, 2000 und 2005.

\begin{tabular}{|c|c|c|c|}
\hline Land & 2000 & 2005 & $\begin{array}{l}\text { Zunahme } \\
\text { in } \%\end{array}$ \\
\hline Schweiz & 416 & 560 & 35 \\
\hline Norwegen & 289 & 547 & 89 \\
\hline Dänemark & 269 & 492 & 83 \\
\hline Frankreich & 262 & 486 & 85 \\
\hline Luxemburg & 249 & 458 & 84 \\
\hline Japan & 452 & 454 & 0 \\
\hline Schweden & 290 & 437 & 51 \\
\hline Italien & 252 & 406 & 61 \\
\hline Finnland & 198 & 404 & 104 \\
\hline Grossbritannien & 212 & 362 & 71 \\
\hline Deutschland* & 216 & 357 & 65 \\
\hline Niederlande & 178 & 310 & 74 \\
\hline Australien & 137 & 267 & 95 \\
\hline Tschechische Republik & 97 & 264 & 172 \\
\hline Portugal & 136 & 251 & 85 \\
\hline Ungarn & 80 & 226 & 183 \\
\hline Südkorea & 121 & 196 & 62 \\
\hline Slowakei & 61 & 133 & 118 \\
\hline Neuseeland & 62 & 112 & 81 \\
\hline Türkei & 34 & 93 & 174 \\
\hline Durchschnitt & 201 & 341 & 70 \\
\hline
\end{tabular}


den Akutkrankenhäusern mit 8,5 Tagen im dritten Rang (wie schon 2000) von 21 Ländern. Abgesehen vom Sonderfall Japan $(19,8$ !) waren die Aufenthalte nur in deutschen Krankenhäusern noch länger $(8,6)$. Die 8,5 Tage Aufent-

\section{Tabelle 4}

Berufstätige Ärzte pro 1000 Einwohner, 2000 und 2005.

\begin{tabular}{|c|c|c|c|}
\hline Land & 2000 & 2005 & Zu- oder Abnahme in \% \\
\hline Belgien & 3,9 & 4 & 2,6 \\
\hline Italien & 4,1 & 3,8 & $-7,3$ \\
\hline Schweiz & 3,5 & 3,8 & 8,6 \\
\hline Spanien & 3,2 & 3,8 & 18,8 \\
\hline Island & 3,4 & 3,7 & 8,8 \\
\hline Niederlande * & 3,2 & 3,7 & 15,6 \\
\hline Norwegen & 2,9 & 3,7 & 27,6 \\
\hline Tschechische Republik & 3,4 & 3,6 & 5,9 \\
\hline Österreich & 3,1 & 3,5 & 12,9 \\
\hline Deutschland & 3,3 & 3,4 & 3,0 \\
\hline Frankreich & 3,3 & 3,4 & 3,0 \\
\hline Portugal * & 3,1 & 3,4 & 9,7 \\
\hline Ungarn & 3,1 & 3 & $-3,2$ \\
\hline Irland * & 2,2 & 2,8 & 27,3 \\
\hline Luxemburg & 2,1 & 2,5 & 19,0 \\
\hline Finnland & 2,3 & 2,4 & 4,3 \\
\hline USA & 2,3 & 2,4 & 4,3 \\
\hline Grossbritannien & 2 & 2,4 & 20,0 \\
\hline Kanada & 2,1 & 2,2 & 4,8 \\
\hline Polen & 2,2 & 2,1 & $-4,5$ \\
\hline Mexiko & 1,6 & 1,8 & 12,5 \\
\hline Südkorea & 1,3 & 1,6 & 23,1 \\
\hline Durchschnitt & 2,8 & 3,0 & 7,2 \\
\hline
\end{tabular}

halt in der Schweiz sind 25\% höher als der OECD-Durchschnitt. Viel kürzere Verweildauern finden sich in Ländern mit ebenfalls guten und gut ausgebauten Akutspitälern.

\section{Zahl der Betten und der Krankenhaustage: durchschnittlich}

Von 2000 bis 2005 hat die Bettenzahl in den Akutspitälern in der Schweiz um 12,2\% abgenommen: von 4,1 auf 3,6. Im OECD-Durchschnitt betrug der Rückgang 6,6\% (von 4,4 auf 4,1 Betten). Die grösste Bettendichte in Europa weisen Deutschland und Österreich mit 6,4 bzw. 6,1 Betten auf. Die USA haben noch 2,7, Schweden hat nur noch 2 Betten je 1000 Einwohner. Die Schweizer Akutspitäler hatten 2005 mit 86,1\% die zweithöchste Bettenbelegung von 17 erfassten Ländern (Durchschnitt: 74,2\%). Im Jahr 2000 betrug sie bei uns $84,8 \%$.

Die OECD verzeichnet für die Schweiz 1,1 Krankenhaustage pro Kopf und Jahr (2005). Dies liegt nahe beim Durchschnitt von 1,2 Tagen für die 17 erfassten Länder. Im Jahr 2000 waren es in der Schweiz noch 1,3 Tage. Diese Abnahme ist die zweitstärkste aller 17 erfassten Länder.

\section{Literatur}

1 OECD Health Data 2007. Statistics and indicators for 30 countries. Paris: OECD Publishing; 2007. CD-ROM mit Benutzerhandbuch, sechssprachig englisch/französisch/deutsch/italienisch/spanisch/ russisch. www.oecd.org/health/healthdata.

2 Bundesamt für Statistik. Prognosen der Kosten des Gesundheitswesens. Neuenburg: BFS; 2007.

3 Marty F. Medikamente. In: Kocher G, Oggier W (Hrsg.). Gesundheitswesen Schweiz 2007-2009. Bern: Hans Huber; 2007. S. 181-92. 